\title{
Composition en acides gras supérieurs \\ de quelques types de fromages consommés en Belgique
}

\author{
par \\ M. VANBELLE, W. VERVACK et M. FOULON \\ (collaboration technique : L. BAILLEUX)
}

\section{INTRODUCTION}

Les fromages entrent pour une part de plus en plus importante dans l'alimentation humaine, où ils constituent une source intéressante en protéines et en matières grasses.

Nous avions étudié précédemment, pour différents types de fromages consommés dans notre pays, le problème de la composition en acides aminés (De Vuyst et al., 1973) et celui de la teneur en lysine disponible (De Vuyst et al., 1972).

Dans le présent travail, nous étudierons plus spécialement la composition de la matière grasse de quelques fromages, en la comparant à la composition en acides gras supérieurs de la graisse butyrique.

Il nous a paru intéressant de voir si la composition en acides gras de la graisse butyrique pouvait subir des variations au cours de la fabrication et de la maturation des fromages.

Cette analyse de la composition en acides gras est actuellement très facilement réalisable par application des techniques de chromatographie en phase gazeuse.

En plus de la détermination des acides gras supérieurs, nous avons étudié simultanément les possibilités de variations de deux

Travail réalisé au Laboratoire de Biochimie de la Nutrition, Directeur : professeur M. VANBelle, Faculté des Sciences Agronomiques de l'Université Catholique de Louvain, place Croix-du-Sud, 3, B-1348 Louvain-la-Neuve (Belgique). 
indices importants caractérisant les matières grasses : l'indice de saponification et l'indice d'iode.

Du point de vue diététique, cette étude présente un intérêt pratique certain, car elle permet de voir l'apport en acides gras polyinsaturés et en acides gras saturés.

L'apport en acides gras polyinsaturés est intéressant à connaître au point de vue diététique, car il permet de pallier certaines maladies, telles que les maladies coronariennes (infarctus du myocarde).

Une récente étude faite en Belgique montre qu'il y a une corrélation entre la consommation de graisse butyrique et la fréquence des maladies coronariennes (Jossens J. V. et al., 1977).

\section{MATERIEL ET METHODES}

\section{A. Matériel}

Nous avons analysé plusieurs sortes de fromages, à raison de six échantillons par type de fromage. Les échantillons ont été prélevés dans le commerce.

Nous avons analysé :

- fromages frais du type Boursin ;

— fromages à pâte ferme cuite : Emmenthal, Gruyère ;

— fromages à pâte ferme non cuite : Chester, Gouda, Ila, Pyrénées ;

— fromages à pâte molle : Brie, Camembert, Herve ;

- Fromages à pâte persillée : Bleu Danois, Roquefort.

Nous avons procédé, pour ces différentes sortes de fromages, aux déterminations suivantes :

1. Indice de saponification.

2. Indice d'iode.

3. Indice d'acides gras libres.

4. Composition en acides gras supérieurs.

D'autres types de fromages ont été analysés en nombre plus réduit d'échantillons (un à quatre) : Hollande, Port-Salut et Tilsitt (fromages à pâte ferme non cuite) ; Bruxelles et Herve fondu (fromages à pâte molle). Nous n'avons déterminé que leur composition en acides gras supérieurs à $\mathrm{C} 8$. 


\section{B. Méthodes analytiques}

La matière grasse est extraite à l'appareil Soxhlet par l'éther sulfurique exempt de peroxyde, celui-ci étant ensuite complètement évaporé.

Sur la matière grasse ainsi obtenue, nous faisons les déterminations suivantes :

— indice de saponification (I.S.) ;

— indice d'iode, par la méthode de Wijs (I.I.) ;

- indice d'acides gras libres, méthode A.O.A.C. (A.O.A.C., 1952) ;

- composition en acides gras supérieurs, après méthylation préalable, par chromatographie en phase gazeuse sur appareil Pye (Cambridge).

TABLEAU 1. - Indices d'iode des différents types de fromages

\begin{tabular}{l|l}
\hline Fromages & Indices d'iode \\
\cline { 2 - 2 } Boursin & $31 \pm 1,00$ \\
Emmenthal & $38 \pm 2,10$ \\
Gruyère & $37 \pm 3,28$ \\
Chester & $33 \pm 2,28$ \\
Gouda & $35 \pm 2,53$ \\
Ila & $30 \pm 0,90$ \\
Pyrénées & $34 \pm 3,39$ \\
Brie & $30 \pm 1,41$ \\
Camembert & $39 \pm 1,41$ \\
Herve & $39 \pm 1,41$ \\
Bleu Danois & $35 \pm 3,22$ \\
Roquefort & $40 \pm 0,89$ \\
& \\
\hline
\end{tabular}


Les conditions analytiques sont les suivantes :

- colonne en borosilicate de $180 \mathrm{~cm}$;

— phase stationnaire : éthylène glycol succinate (15 p. 100) ;

- support interne : chromosorb W 80 - 100 mesh (85 p. 100);

- température du four $\left(185^{\circ} \mathrm{C}\right)$;

- débit d'azote $(60 \mathrm{ml} / \mathrm{mn})$;

- détecteur à ionisation de flamme (F.I.D.) ;

- enregistreur Sefram Servotrace, vitesse de déroulement du papier et sensibilité sont variables selon les acides gras analysés.

La méthode utilisée pour la détermination des acides gras est celle de la Station de Recherches Laitières de Gembloux (Guyot A. et Piraux E., 1964).

TABLEAU 2. - Indices de saponification des différents types de fromages

\begin{tabular}{l|l}
\hline Fromages & $\begin{array}{c}\text { Indices de } \\
\text { saponification }\end{array}$ \\
\hline Boursin & $227 \pm 3,43$ \\
Emmenthal & $222 \pm 3,40$ \\
Gruyère & $220 \pm 3,63$ \\
Chester & $227 \pm 6,84$ \\
Gouda & $229 \pm 4,47$ \\
Ila & $202 \pm 5,15$ \\
Pyrénées & $206 \pm 9,53$ \\
Brie & $222 \pm 8,93$ \\
Camembert & $224 \pm 6,19$ \\
Herve & $250 \pm 5,63$ \\
Bleu Danois & $211 \pm 6,04$ \\
Roquefort & $212 \pm 6,78$ \\
\hline
\end{tabular}




\section{RESULTATS EXPERIMENTAUX}

Nous avons rassemblé les résultats expérimentaux dans les tableaux suivants :

1. Indices d'iode des différents types de fromages.

2. Indices de saponification.

3. Indices d'acides gras libres.

4. Composition en acides gras, supérieurs à $\mathrm{C}_{8}$, de la matière grasse des différents fromages.

Dans ce dernier tableau, deux pics non identifiés $\mathrm{x}$ et $\mathrm{y}$, situés entre $C_{14}$ et $C_{16}$ et correspondant vraisemblablement à l'ensemble iso $C_{15}$, $C_{15}, C_{15: 2}$ et $C_{16}$ ont été surfacés et regroupés.

TABLEAU 3. - Indices d'acidité libre des différents types de fromages

\begin{tabular}{l|c}
\hline Fromages & $\begin{array}{c}\text { Indices d'acidité } \\
\text { libre }\end{array}$ \\
\hline Boursin & $2,69 \pm 0,79$ \\
Emmenthal & $8,73 \pm 0,62$ \\
Gruyère & $4,29 \pm 0,38$ \\
Chester & $4,70 \pm 0,70$ \\
Gouda & $3,44 \pm 0,28$ \\
Ila & $5,10 \pm 1,45$ \\
Pyrénées & $4,20 \pm 2,76$ \\
Brie & $5,98 \pm 0,87$ \\
Camembert & $12,47 \pm 7,69$ \\
Herve & $9,34 \pm 4,79$ \\
Bleu Danois & $25,39 \pm 8,36$ \\
Roquefort & $15,43 \pm 6,81$ \\
& \\
\hline
\end{tabular}


TABLEAU 4

Composition en acides gras supérieurs de quelques types de fromages consommés en Belgique

\begin{tabular}{|c|c|c|c|c|c|c|c|}
\hline & \multirow{2}{*}{\multicolumn{2}{|c|}{$\begin{array}{c}\text { Fromages frais } \\
\text { Boursin }\end{array}$}} & \multicolumn{5}{|c|}{ Fromages à pâte ferme cuite } \\
\hline & & & \multicolumn{2}{|c|}{ Emmenthal } & \multicolumn{3}{|c|}{ Gruyère } \\
\hline & Moyenne & $\begin{array}{l}\text { Déviation } \\
\text { standard }\end{array}$ & Moyenne & $\begin{array}{l}\text { Déviation } \\
\text { standard }\end{array}$ & Moyenne & $\begin{array}{l}\mathrm{I} \\
\mathrm{s}\end{array}$ & $\begin{array}{l}\text { Déviation } \\
\text { standard }\end{array}$ \\
\hline $\begin{array}{l}\text { p. } 100 \text { matière sèche } \\
\text { p. } 100 \text { matière grasse }\end{array}$ & $\begin{array}{l}53,62= \\
39,67=\end{array}$ & $\begin{array}{l} \pm 2,35 \\
\pm \quad 1,10\end{array}$ & $\begin{array}{l}66,84 \pm \\
31,30 \pm\end{array}$ & $\begin{array}{l} \pm \quad 4,52 \\
\pm \quad 2,16\end{array}$ & $\begin{array}{l}65,37= \\
31,65\end{array}$ & \pm & $\begin{array}{l}1,90 \\
2,19\end{array}$ \\
\hline $\begin{array}{cc}\text { Acides gras } & \mathrm{C}_{8} \\
& \mathrm{C}_{10} \\
& \mathrm{C}_{12} \\
& \mathrm{C}_{14} \\
& \mathrm{XY} \\
\mathrm{C}_{18} \\
\mathrm{C}_{18: 1} \\
\mathrm{C}_{18} \\
\mathrm{C}_{18: 1} \\
\mathrm{C}_{18: 2} \\
\mathrm{C}_{18: 3}\end{array}$ & $\begin{aligned} 0,90 & = \\
2,53 & = \\
3,69 & = \\
12,55 & = \\
3,73 & = \\
36,64 & = \\
2,29 & = \\
10,55 & = \\
24,25 & = \\
1,13 & = \\
1,72 & =\end{aligned}$ & $\begin{array}{ll} \pm & 0,15 \\
\pm & 0,18 \\
\pm & 0,38 \\
\pm & 1,06 \\
\pm & 0,70 \\
\pm & 1,28 \\
\pm & 0,19 \\
\pm & 0,97 \\
\pm & 0,98 \\
\pm & 0,22 \\
\pm & 0,71\end{array}$ & $\begin{aligned} 0,71 & \pm \\
1,78 & \pm \\
2,76 & \pm \\
9,22 & \pm \\
3,06 & \pm \\
29,05 & \pm \\
1,56 & \pm \\
14,40 & \pm \\
30,45 & \pm \\
2,04 & \pm \\
4,95 & \pm\end{aligned}$ & $\begin{array}{ll} \pm & 0,41 \\
\pm & 0,53 \\
\pm & 1,54 \\
\pm & 0,91 \\
\pm & 0,43 \\
\pm & 3,33 \\
\pm \quad & 0,44 \\
\pm & 2.21 \\
\pm & 2,37 \\
\pm & 0,96 \\
\pm & 1,52\end{array}$ & $\begin{aligned} 0,93 & = \\
2,22 & = \\
3,09 & = \\
11,60 & = \\
2,97 & = \\
29,86 & = \\
2,20 & = \\
11,32 & = \\
30,84 & = \\
1,75 & = \\
3,23 & =\end{aligned}$ & $\begin{array}{l} \pm \\
\pm \\
\pm \\
\pm \\
\pm \\
\pm \\
\pm \\
\pm \\
\pm \\
\pm \\
\pm\end{array}$ & $\begin{array}{l}0,37 \\
0,40 \\
0,68 \\
1,34 \\
0,69 \\
4,33 \\
2,23 \\
2,94 \\
4,17 \\
1,04 \\
1,79\end{array}$ \\
\hline$\frac{\text { Insaturés }}{\text { Saturés }}$ & $0,42=$ & $\pm 0,02$ & $0,64 \pm$ & $\pm 0,09$ & $0,62=$ & \pm & 0,14 \\
\hline$\frac{\text { Polyinsaturés }}{\text { Saturés }}$ & $0,05=$ & $\pm 0,01$ & $0,12 \pm$ & $\pm 0,04$ & $0,08=$ & \pm & 0,05 \\
\hline Nombre d'échantillons & & 6 & & 6 & & 7 & \\
\hline
\end{tabular}


TABLEAU 4 (suite 1). - Composition en acides gras supérieurs de quelques types de fromages consommés en Belgique

Fromages à pâte ferme non cuite

p. 100

p. 100

matière grasse

Acides gras $\mathrm{C}_{\mathrm{s}}$

$$
\begin{array}{l|l}
\mathrm{C}_{8} & 0,99 \pm 0,36 \\
\mathrm{C}_{10} & 2,31 \pm 0,57
\end{array}
$$

$\mathrm{C}_{10} \quad 2,31 \pm 0,57$

\begin{tabular}{l|r}
$\mathrm{C}_{12}$ & $2,81 \pm 0,89$ \\
$\mathrm{C}_{14}$ & $11,34 \pm 1,54$ \\
$\mathrm{XY}$ & $3,22 \pm 0,43$
\end{tabular}

$\mathrm{XY} \quad 3,22 \pm 0,43$

\begin{tabular}{ll}
$\mathrm{C}_{16}$ & $29,75 \pm 3,08$ \\
\hline
\end{tabular}

$\mathrm{C}_{16: 1} \quad 1,66 \pm 1,03$

$\mathrm{C}_{18} \quad 14,43 \pm 1,95$

$\mathrm{C}_{18: 1} \quad 28,40 \pm 3,56$

$\begin{array}{ll}\mathrm{C}_{18}: 2 & 2,48 \pm 2,73 \\ \mathrm{C}_{1}: 2,60 \pm 2,64\end{array}$

$\mathrm{C}_{18: 3}$

$2,60 \pm 2,64$

Insaturés

Saturés

Polyinsaturés

Saturés

Nombre

d'échantillons

$$
0,55 \pm 0,11
$$

$0,08 \pm 0,10$

10 matière sèche

Fromages à pâte ferme non cuite

N.B. : $\mathrm{XY}=$ ensemble iso $\mathrm{C}_{15}, \mathrm{C}_{15}, \mathrm{C}_{15: 2}$ et iso $\mathrm{C}_{16}$

\begin{tabular}{|c|c|} 
Chester fondu & Gouda \\
$\begin{array}{c}\text { Moyen- Dévia- } \\
\text { tion } \\
\text { ne } \\
\text { stan- } \\
\text { dard }\end{array}$ & $\begin{array}{c}\text { Moyen- Dévia- } \\
\text { tion } \\
\text { ne }\end{array}$ \\
stan- \\
dard
\end{tabular} \mid

\section{Hollande}

Moyen- Dévia-

ne tion
stan-

dard

$61,08 \pm 2,80$

$25,93 \pm 3,16$

$0,74 \pm 0,40$

$2,97 \pm 0,76$

$10,15 \pm 1,08$

$2,95 \pm 0,24$

$30,60 \pm 2,58$

$3,43 \pm 1,63$

$12,23 \pm 1,89$

$30,88 \pm 3,44$

$1,56 \pm 1,07$

$2,35 \pm 1,19$

$0,62 \pm 0,09$

0,61

0,05

$0,07 \pm 0,03$

0,05 dard

- -

14,58

5,13

26,35

3,17
12,82

12,82

26,99

1,70

1,44

0,50
$2,16 \pm 0,69$

\begin{tabular}{c|c} 
Ila \\
Moyen- Dévia-
\end{tabular}

ne tion

$-\frac{1}{20}$

$1,02-0,58 \pm 0,10$

$\begin{array}{lll}1,02 & - & 0 \\ 2,49 & - & 1,59 \pm 0,22\end{array}$

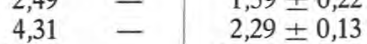

$2,29 \pm 0,13$
$10,28 \pm 0,68$

$3,60 \pm 0,67$

$31,73 \pm 1,54$

$2,70 \pm 0,43$

$13,88 \pm 0,94$

$30,31 \pm 2,68$

$1,59 \pm 0,24$

$1,44 \pm 0,43$

$0,57 \pm 0,07$

$0,57 \pm 0,07$

$0,05 \pm 0,01$

$0,04 \pm 0,02$

$0,06 \pm 0,03$

$0,11 \quad-$ 
TABLEAU 4 (suite 2). - Composition en acides gras supérieurs de quelques types de fromages consommés en Belgique

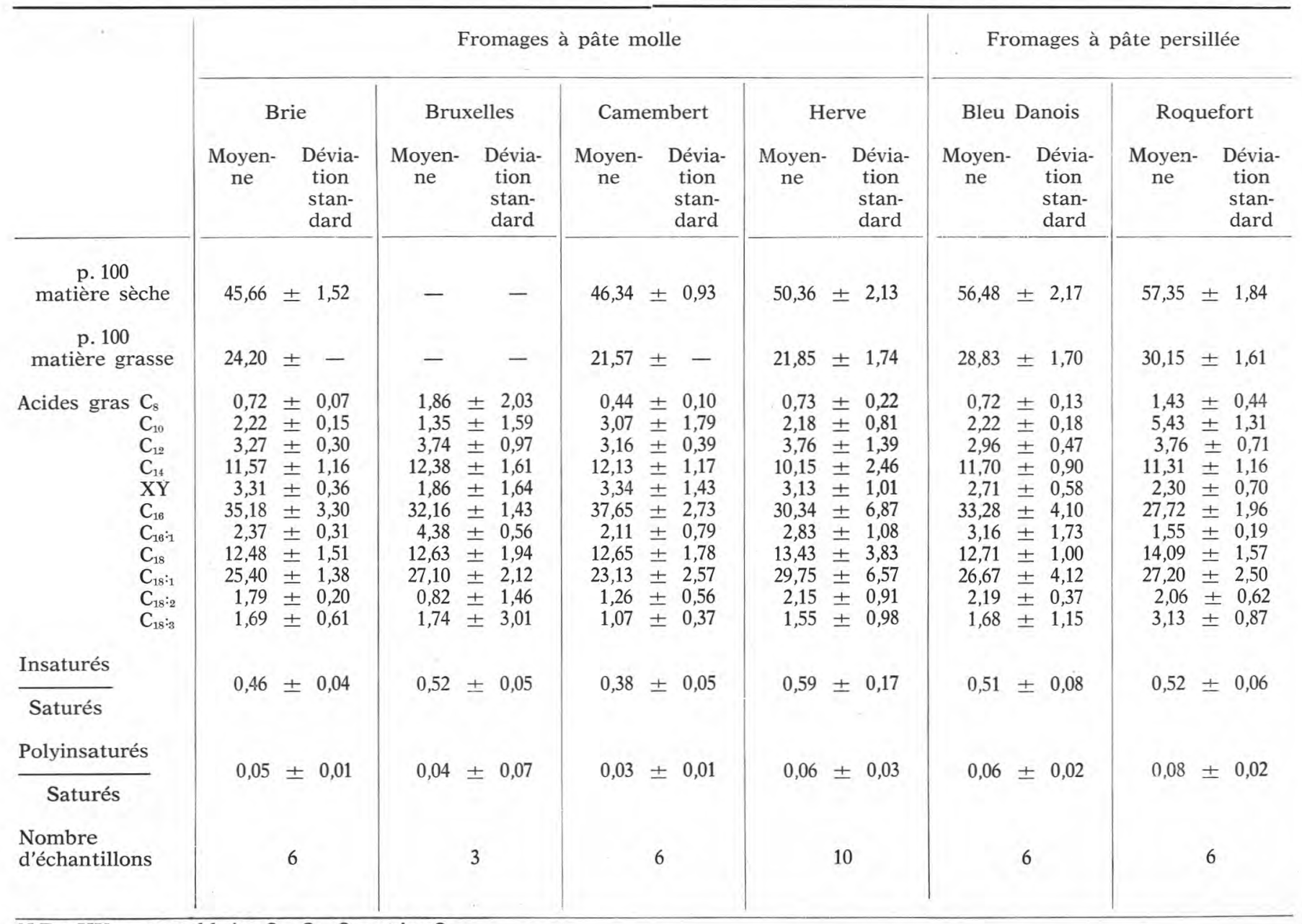




\section{DISCUSSION DES RESULTATS}

\section{Indice d'iode}

Ne disposant pas du lait, et donc de la matière grasse ayant servi à la fabrication des divers types de fromages étudiés, nous avons repris dans la littérature des données relatives à la graisse butyrique.

Williams K.A. (1966), renseigne des valeurs oscillant entre 26 et 40, tandis que Wolff J.P. (1968), renseigne des chiffres variant entre 33 et 45 .

Au Danemark, on adopte 38 comme indice d'iode, contre 37 en France, 42 en Irlande et 38 en Nouvelle-Zélande.

De l'ensemble de ces données, on peut dire que l'indice d'iode de la graisse butyrique peut osciller entre les valeurs extrêmes suivantes :

\section{Indice d'iode: de 26 à 45}

Pour l'ensemble des fromages analysés, nous trouvons des chiffres variant entre les limites suivantes :

\section{Indice d'iode: de 30 à 40}

Les chiffres d'indices d'iode des fromages se situent donc dans des limites communément acceptées pour la graisse butyrique.

La fabrication du fromage n'a donc pas altéré sensiblement l'indice d'iode de la graisse butyrique.

\section{Indice de saponification}

Williams K.A. (1966) renseigne des valeurs oscillant entre 215 et 235. Wolff J. P. (1968), pour sa part, renseigne des valeurs comprises entre 226 et 232 .

L'A.O.A.C. (1952) admet une limite inférieure de 210.

En résumé, on peut dire que l'indice de saponification de la graisse butyrique peut varier entre les limites extrêmes suivantes :

Indice de saponification: de 210 à 235

Pour les fromages, nous trouvons des indices oscillant entre les valeurs suivantes:

Indice de saponification: de 202 à 250 
Certains fromages ont donc un indice de saponification légèrement inférieur à la limite inférieure donnée pour la graisse butyrique, d'autres ont un chiffre un peu supérieur à la limite supérieure renseignée.

La plus grande partie des fromages analysés présente un indice de saponification se situant dans les limites renseignées dans la littérature pour la graisse butyrique.

Comme pour l'indice d'iode, la fabrication du fromage ne semble pas altérer sensiblement l'indice de saponification.

\section{Indice d'acidité libre}

Nous n'avons trouvé dans la littérature (Wolff J.P., 1968) que des données variant entre 0,8 et $1,4 \mathrm{mg}$ de $\mathrm{KOH}$ par gramme de matière grasse butyrique. tantes.

Pour les fromages, nous constatons des variations plus impor-

Le fromage du type Boursin a le plus petit indice d'acidité libre soit $2,69 \pm 0,79$, alors que pour la graisse butyrique pure, le chiffre maximum renseigné est de 1,4 .

Tous les autres fromages se signalent par des indices d'acidité libre plus élevés, pouvant aller jusque 15,43 $\pm 6,81$ pour le Roquefort, et même jusque 25,39 $\pm 8,36$, pour le Bleu Danois.

L'augmentation de cet indice est le signe du degré d'hydrolyse plus ou moins prononcé de la matière grasse des fromages, suite aux transformations qu'elle subit au cours de la préparation des fromages.

\section{Composition en acides gras supérieurs à $C_{s}$}

Comme le montre le tableau 5 qui suit, la composition en acides gras de la graisse butyrique semble essentiellement variable.

Elle varie en fonction de la saison, de l'alimentation, de la race, de la région...

On voit donc que, a priori, on peut s'attendre à avoir de grandes variations dans la composition en acides gras des fromages.

Le tableau 4, donnant la composition en acides gras supérieurs à $C_{s}$ des différents fromages analysés, nous montre néanmoins que les teneurs relatives de ces acides gras restent assez constantes, quel que soit le type de fromage. 
En effet, compte tenu des compositions variables des acides gras de la graisse butyrique suivant la saison par exemple (tab. 5), les rapports $\frac{\text { acides gras insaturés }}{\text { acides gras saturés }}$ restent, pour les différents types de fromages, comparables aux valeurs déterminées par Manwaery (1972) sur la graisse butyrique d'été et d'hiver (respectivement 0,59 et 0,44$)$.

TABLEAU 5. - Composition en acides gras de la graisse butyrique

\begin{tabular}{|c|c|c|c|c|}
\hline \multirow{2}{*}{ Acides gras } & \multicolumn{2}{|c|}{ Wolff J. P., 1968} & \multicolumn{2}{|c|}{ Manwaery, 1972} \\
\hline & minimum & maximum & été & hiver \\
\hline $\mathrm{C}_{4}$ & 2,8 & 5,0 & 3,65 & 3,75 \\
\hline $\mathrm{C}_{6}$ & 1,1 & 3,0 & 2,10 & 2,30 \\
\hline $\mathrm{C}_{8}$ & 1,0 & 2,7 & 1,25 & 1,39 \\
\hline $\mathrm{C}_{10}$ & 2,1 & 3,9 & 2,57 & 2,98 \\
\hline $\mathrm{C}_{12}$ & 2,6 & 4,2 & 2,92 & 3,44 \\
\hline $\mathrm{C}_{14}$ & 8,2 & 14,5 & 9,52 & 10,78 \\
\hline $\mathrm{C}_{16}$ & 22,0 & 37,5 & 22,49 & 28,42 \\
\hline $\mathrm{C}_{18}$ & 6,6 & 13,5 & 12,20 & 9,52 \\
\hline $\mathrm{C}_{18: 1}$ & 16,2 & 34,5 & 29,61 & 24,38 \\
\hline $\mathrm{C}_{18: 2}$ & 1,3 & 2,9 & 1,74 & 1,81 \\
\hline $\mathrm{C}_{18: 3}$ & 0,7 & 4,8 & 2,37 & 1,13 \\
\hline$\frac{\text { P.I. }}{\mathrm{S}}$ & & & 0,07 & 0,05 \\
\hline
\end{tabular}

La graisse butyrique est donc caractérisée par une très faible teneur en acides gras polyinsaturés (acides gras essentiels) et un rapport Acides gras polyinsaturés

Acides gras saturés 
Pour l'ensemble des fromages analysés, nous trouvons en effet des chiffres variant entre les limites suivantes:

$$
0,38 \text { et } 0,64
$$

De même, il n'existe aucune différence significative entre la graisse butyrique et les différents types de fromages étudiés en ce qui concerne le rapport $\frac{\text { acides gras polyinsaturés }}{\text { acides gras saturés }}$.

Manwaery (1972) détermina les valeurs de 0,07 et 0,05 pour les graisses butyriques d'été et d'hiver.

Pour l'ensemble des fromages analysés, nous trouvons des chiffres variant entre les limites suivantes :

$$
0,03 \text { et } 0,12
$$

Il s'ensuit que la graisse butyrique n'a pas subi de variations significatives lors de la fabrication des différents fromages, en ce qui concerne sa composition en acides gras supérieurs à $\mathrm{C}_{8}$.

\section{Matière sèche et matière grasse}

$\mathrm{Au}$ sein d'une même catégorie de fromages, il est également intéressant de constater que les teneurs en matière sèche et en matière grasse restent, elles aussi, assez constantes.

\section{a) Matière sèche}

Du tableau 4, nous pouvons déduire, pour les différentes catégories de fromages étudiées, les teneurs extrêmes suivantes :

- Fromages frais

54 p. 100

- Fromages à pâte ferme non cuite

65 à 66 p. 100

- Fromages à pâte ferme cuite

55 à 63 p. 100

- Fromages à pâte molle 45 à 50 p. 100

- Fromages à pâte persillée 56 à 57 p. 100

b) Matière grasse

De même, nous avons déterminé les teneurs suivantes en matière grasse :

- Fromages frais

- Fromages à pâte ferme non cuite

- Fromages à pâte ferme cuite

- Fromages à pâte molle

- Fromages à pâte persillée
40 p. 100

31 p. 100

22 à 33 p. 100

21 à 24 p. 100

29 à 30 p. 100 


\section{CONCLUSIONS}

La graisse butyrique ne subit pas de variations significatives au cours de la fabrication des fromages, au moins pour ce qui est des acides gras supérieurs à $\mathrm{C}_{\mathrm{s}}$.

Nous nous sommes limités à l'analyse des acides gras supérieurs à $C_{s}$ parce que, lors de la méthylation, il existe des risques réels de pertes, les acides gras inférieurs à $\mathrm{C}_{\mathrm{s}}$, s'ils sont méthylés, ont un point d'ébullition trop bas.

Pour ce qui est des indices d'iode de la matière grasse des fromages, ils se situent dans les limites données par la littérature pour la graisse butyrique. L'indice d'iode des fromages varie entre les limites de 30 à 40 .

Pour ce qui est des indices de saponification, ils varient pour le plus grand nombre de fromages entre 220 et 229 . Il y a cinq exceptions : Pyrénées (206), Ila (202), Herve (250), Roquefort (212) et Bleu Danois (211).

Selon la littérature, les oscillations sont comprises entre les valeurs 215 et 235 pour la graisse butyrique pure.

L'indice d'acidité libre est plus élevé pour la matière grasse des fromages, que pour la graisse butyrique pure.

Le Boursin (fromage blanc) a un indice d'acidité libre de 2,69 et le Bleu Danois a un indice d'acidité libre de 25,39, chiffre le plus élevé que nous ayons trouvé.

Pour la graisse butyrique pure, il est au minimum de 0,8 et au maximum de 1,4 .

Cette augmentation d'acidité libre chez les fromages, est à mettre au compte de la lypolyse, des dégradations d'acides aminés pour donner des acides gras libres à courtes chaînes, et des fermentations en général.

Quant au rapport polyinsaturés/saturés, il oscille, pour l'ensemble des fromages, entre un minimum de 0,05 et un maximum de 0,12. Ce même rapport varie entre 0,05 et 0,07 pour la graisse butyrique pure.

Le rapport acides gras polyinsaturés/acides gras saturés idéal étant de 2, il s'ensuit que du point de vue diététique, le beurre et la matière grasse des fromages sont très loin de ce rapport idéal.

Ce point a été mis en évidence dans un récent article (Joossens J.V. et al., 1977). 


\section{R é s u m é}

Dans le présent travail, les auteurs ont étudié la qualité de la matière grasse de différents fromages couramment consommés en Belgique.

Ils en ont déterminé les indices d'iode, de saponification et d'acidité libre ainsi que leur composition en acides gras supérieurs à $\mathrm{C}_{\text {s. }}$.

Ils arrivent à la conclusion que les processus de fabrication n'ont pas sensiblement altéré la composition de la matière grasse par rapport à la graisse butyrique pure, sauf pour ce qui est de l'indice d'acidité libre, qui augmente fortement pour l'ensemble des fromages.

Au point de vue du rapport acides gras polyinsaturés/acides gras saturés, il oscille entre les valeurs limites de $0,05-0,12$ pour les fromages, et de 0,05-0,07 pour la graisse butyrique pure.

Du point de vue diététique, ce rapport devrait être de 2 ou proche de 2 pour éviter les incidents cardiaques dus à un apport insuffisant d'acides gras polyinsaturés.

\section{S u m mary}

In the present paper, the authors studied the quality of the fat from many different cheeses: iodine number, saponification number, free fatty acids number and also the fatty acid composition (fatty acids higher than $C_{8}$ ) have been determinated.

They concluded that the industrial processes of fabrication have not modified the composition of cheese fat versus butyric fat, except for the free fatty acid number, who is greetly extended.

On the point of vue of the ratio polyunsaturated fatty acids/saturated fatty acids who is between $0,05-0,12(0,05-0,07$ for butterfat $)$, this ratio is far from the ideal dietetic ratio 2 . 


\section{Bibliographie consultée}

A.O.A.C. Methods of Analysis (1952). - Association of Official Analytical Chemists. P.O. Box 540 Benjamin Franklin Station. Washington DC 20044.

De Vuyst (A.), Vervack (W.), Charlier (H.) et JadiN (V.) (1972). - La teneur en lysine totale et disponible du lait, des fromages et des produits de boulangerie ayant subi un traitement industriel. Le Lait, 52, 517, 444.

De Vuyst (A.), Vervack (W.), Vanbelle (M.) et Foulon (M.) (1973). - La composition en acides aminés de quelques fromages courants. Le Lait, 53, 529-530, 625.

GuYot (A.) et Piraux (E.) (1964). - Etude par la chromatographie en phase gazeuse de la matière grasse des beurres de laiterie belges. Bulletin de l'Institut Agronomique et des Stations de Recherches de Gembloux, tome XXXII, $\mathrm{n}^{\circ} 2$.

Joossens (J. V.) et al. (1977). - The Lancet, 1069-1072.

Williams (K. A.) (1966). - Gils, Fats and Fatty Foods. Fourth Edition, 133-177, Churchill Jond A End, London.

WoLfF (J. P.) (1968). — Manuel d'analyse des corps gras, 114-120, Azoulay Ed., Paris. 